\title{
Synthesis, Characterization and Antimicrobial Activity of 5-Substituted indole-2,3-dione Based 4 -Thiazolidione Derivatives
}

\section{A. ZAHIR HUSSAIN ${ }^{1}$, M. NAGOOR MEERAN ${ }^{2 *}$ and A. SANKAR ${ }^{3}$}

${ }^{1} \mathrm{PG}$ and Research Department of Chemistry, Jamal Mohamed College, Trichy, India

${ }^{2} \mathrm{PG}$ and Research Department of Chemistry, Vivekanandha College of Arts and Sciences for Women (Autonomous), Tiruchengode, India

${ }^{3}$ Department of Chemistry, Kandaswami Kandar's College, P. Velur, Namakkal, India nagoorjmc@ rediffmail.com

Received 20 December 2015 / Accepted 31 December 2015

\begin{abstract}
A series of 5-substituted indole- 2,3-dione based spiro-4-thiazolidiones were synthesized, characterization and evaluated for their antimicrobial activity. Condensation of 5-substituted indole-2,3dione with substituted primary aryl amine was formed series of Schiff bases (1) which on reaction with thioglycolic acid and thiolactic acid in 1,4-dioxane afforded the formation of the corresponding 4thiazolidinones $(\mathbf{2}, \mathbf{3})$. All the synthesized compounds were characterized on the basis of their IR, ${ }^{1} \mathrm{H}$ and ${ }^{13} \mathrm{C}$ NMR and elemental analysis. The antimicrobial activity of all the compounds (D01-D04, E01-E04) showed significant activity against the selected micro organisms.
\end{abstract}

Keywords: 5-Substituted indole -2,3-dione, Spiro-4-thiazolidiones, Antimicrobial

\section{Introduction}

Spirocyclic system containing one carbon atom common to two rings are structurally interesting ${ }^{1}$. Spiro compounds represent an important class of naturally occurring substances and their characteristic is the highly biological properties ${ }^{2,3} .1-H$-indole-2,3-dione, (Isatin) and derivatives possess a broad range of biological and pharmacological properties and are widely used as starting materials for the synthesis of a broad range of heterocyclic compounds and substrates for drug synthesis ${ }^{4}$. It was first prepared by Erdmann and Laurent through the oxidation of indigo by nitric acid and chromic acid ${ }^{5,6}$. Some of its derivatives specifically Haloisatin and Nitroisatin show a wide range of biological and pharmacological activities such as antimicrobial ${ }^{7-12}$, anticonvulsant ${ }^{13,14}$, analgesic ${ }^{15,16}$, anticancer ${ }^{17,18}$, antitubercular $^{19}$, antiviral ${ }^{20-22}$ and anti-HIV ${ }^{23}$ activities. The literatures survey revealed that introduction of electron withdrawing groups at positions 5, 6 and 7 greatly increased the activities of isatin, with substitution at the $5^{\text {th }}$ position is being most favorable. 4-Thiazolidinones also possess various important biological activities such as antibacterial, 
antifungal, antiviral, diuretic, antituberculostatic, anti-HIV, antihistaminic, anticancer, anticonvulsant, anti inflammatory and analgesic properties ${ }^{24-26}$. Spiro heterocyclic compounds including thiazolidine moiety have antimicrobial activity ${ }^{27}$. Spiroindole heterocyclic, in which the indole ring is linked to the other heterocyclic system through the spirocarbon atom at C-3, show an increased spectrum of biological activities. Systematic investigations of this class of heterocyclic have been carried out by Joshi and co-workers ${ }^{28}$.

The synthesis, characterization and antimicrobial evaluation of 5-substituted indole-2,3dione based spiro-4-thiazolidiones have been undertaken as per the scheme mentioned in the experimental section. All the synthesized compounds were characterized on the basis of their physical properties IR, ${ }^{1} \mathrm{H}$ and ${ }^{13} \mathrm{C}$ NMR spectral data and elemental analysis.

\section{Experimental}

The melting points were carried out in open capillary tube and were uncorrected. Thin layer chromatography was performed using silica gel coated on a glass plate and spots were visualized by exposure to iodine vapour. IR spectra of compounds were scanned on Shimadzu IR spectrophotometer using $\mathrm{KBr}$ disc and expressed in $\mathrm{cm}^{-1} .{ }^{1} \mathrm{H}$ and ${ }^{13} \mathrm{C} \mathrm{NMR}$ spectra were recorded in DMSO- $\mathrm{D}_{6}$ on BRUKER spectrometer using TMS as an internal standard (chemical shifts in $\delta, \mathrm{ppm}$ ). The elemental analysis values of $\mathrm{C}, \mathrm{H}, \mathrm{N}$ and $\mathrm{S}$ are in good agreement with the calculated values. The 5-substituted Indole-2,3-dione based Schiff bases ${ }^{29}$ were prepared from 2-benzoyl-4-chloroaniline in Scheme 1. The synthesis of the target compounds was accomplished according to the reaction sequence illustrated in Scheme 2.<smiles>[Y]C(=O)O[N+](=O)[O-]</smiles>

\section{Scheme 1}

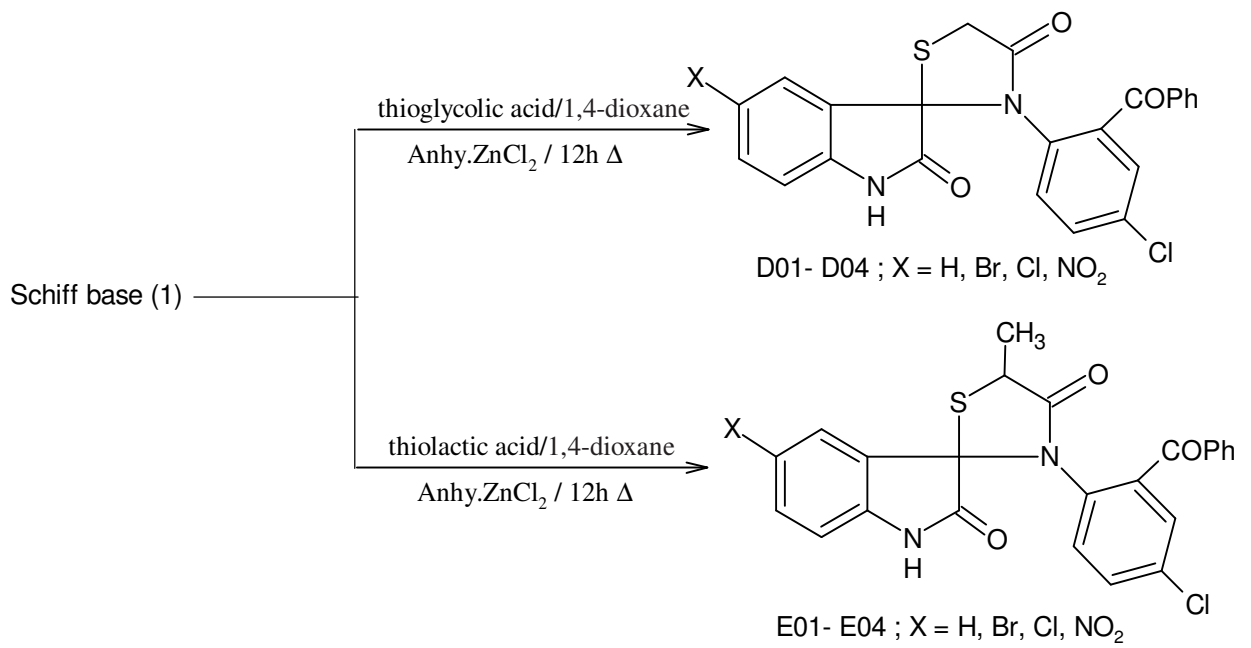

Scheme 2 
Synthesis of 5-substituted indol-2,3-dione based spiro-4-thiazolidiones ${ }^{30}$ (D01-D04)

A mixture of Schiff bases ${ }^{29}$ (1) $(0.01 \mathrm{~mol})$ and thioglycolic acid $(0.01 \mathrm{~mol})$ was refluxed with 1,4-dioxane for $12 \mathrm{~h}$ in the presence of zinc chloride. The completion of reaction was monitored by TLC (Pet ether: ethyl acetate, 3:2). After completion, reaction mixture was poured in ice cold water. The product formed was isolated washed with water and recrystallized from ethanol to give compound 2(D01). Similarly other compounds D02-D04 were synthesized using same procedure.

\section{Synthesis of 5-substituted indol-2,3-dione based 5'-methyl-spiro-4-thiazolidiones ${ }^{30}$ (E01-E04)}

A mixture of Schiff bases ${ }^{29}$ (1) $(0.01 \mathrm{~mol})$ and thiolactic acid $(0.01 \mathrm{~mol})$ was refluxed with 1,4-dioxane for $12 \mathrm{~h}$ in the presence of zinc chloride. The completion of reaction was monitored by TLC (Pet ether: ethyl acetate, 3:1.5). After completion, reaction mixture was poured in ice cold water. The product formed was isolated washed with water and recrystallized from ethanol to give compound 3(E01). Similarly other compounds of E02-E04 were synthesized using same procedure.

\section{Results and Discussion}

Synthesis of Schiff base was done as mentioned in scheme 1. The required starting material of Schiff base (A01-A04) was already confirmed by reported method ${ }^{29}$. It was further on treatment with thioglycolic acid and thiolactic acid yielded the 4thiazolidinones (D01-D04; E01-E04). The spectral analysis of all the compounds was done by IR, ${ }^{1} \mathrm{H}$ and ${ }^{13} \mathrm{C}$ NMR and the spectral data were consistent with the assigned structures. Figure (D01-D04, E01-E04)<smiles>O=C(Oc1ccccc1)c1cc(Cl)ccc1N1C(=O)CSC12C(=O)Nc1ccccc12</smiles><smiles>O=C(c1ccccc1)c1cc(Cl)ccc1N1C(=O)CSC12C(=O)Nc1ccc(Br)cc12</smiles><smiles>O=C(c1ccccc1)c1cc(Cl)ccc1N1C(=O)CSC12C(=O)Nc1ccc(Cl)cc12</smiles><smiles>O=C(c1ccccc1)c1cc(Cl)ccc1N1C(=O)CSC12C(=O)Nc1ccc([N+](=O)[O-])cc12</smiles><smiles>CC1SC2(C(=O)Nc3ccccc32)N(c2ccc(Cl)cc2C(=O)c2ccccc2)C1=O</smiles><smiles>CCOC(=O)c1ccc(N2C(=O)C(C)SC23C(=O)Nc2ccc(Br)cc23)c(C(=O)c2ccccc2)c1</smiles> 

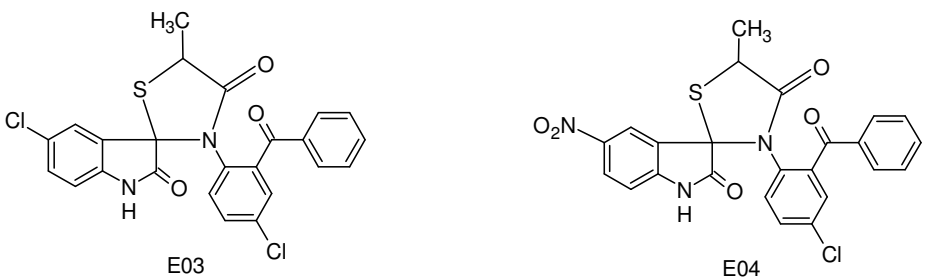

3'-(2-Benzoyl-4-chlorophenyl)-4'H-spiro[indole-3,2'-[1,3]thiazolidine]-2,4'(1H)-

dione; (D01)

M.p: $203{ }^{\circ} \mathrm{C}$; IR (KBr) $\lambda_{\max }$ in $\mathrm{cm}^{-1}: 3417$ (N-H str), 3062 (Ar C-H str), 2924 (Ali C-H Str), 1705 (C=O str), 1680 (Spiro $\mathrm{C}=\mathrm{O}$ ), 1612 (N-C=O str), 736 (C-S str); ${ }^{1} \mathrm{H}-\mathrm{NMR}$ (DMSO-d ${ }_{6}$, $400 \mathrm{MHz}) \delta: 11.57(\mathrm{~s}, 1 \mathrm{H}), 7.74-7.70(\mathrm{t}, J=7.2 \mathrm{~Hz}, 1 \mathrm{H}), 7.64-7.63(\mathrm{~d}, J=7.6 \mathrm{~Hz}, 1 \mathrm{H}), 7.51-$ $7.40(\mathrm{~m}, 5 \mathrm{H}), 7.33-7.30(\mathrm{dd}, J=8.8 \mathrm{~Hz}, 2.4 \mathrm{~Hz}, 1 \mathrm{H}), 7.28-7.26(\mathrm{~d}, J=7.6 \mathrm{~Hz}, 1 \mathrm{H}), 7.19-7.18$ $(\mathrm{d}, J=2.4 \mathrm{~Hz}, 1 \mathrm{H}), 7.08-7.05(\mathrm{t}, J=7.6 \mathrm{~Hz}, 1 \mathrm{H}), 6.92-6.90(\mathrm{~d}, J=9.2 \mathrm{~Hz}, 1 \mathrm{H}), 3.868(\mathrm{~s}, 2 \mathrm{H})$; ${ }^{13}$ C-NMR (DMSO-d $\left.6,100 \mathrm{MHz}\right) \delta: 196,184,174,110-141,77,35$; Anal. Found: C, 63.52; $\mathrm{H}, 3.48 ; \mathrm{N}, 6.44 ; \mathrm{S}, 7.36(\%)$. Calc. for $\left(\mathrm{C}_{23} \mathrm{H}_{15} \mathrm{ClN}_{2} \mathrm{O}_{3} \mathrm{~S}\right): \mathrm{C}, 63.46 ; \mathrm{H}, 3.44 ; \mathrm{N}, 6.43 ; \mathrm{S}$, 7.37(\%).

3'-(2-Benzoyl-4-chlorophenyl)-5-bromo-4'H-spiro[indole-3,2'-[1,3]thiazolidine]2,4'(1H)-dione; (D02)

M.p: $201{ }^{\circ} \mathrm{C}$; IR (KBr) $\lambda_{\max }$ in $\mathrm{cm}^{-1}: 3417$ (N-H Str), 3062 (Ar C-H Str), 2962 (Ali C-H Str), $1697(\mathrm{C}=\mathrm{O}$ str), 1671 (Spiro $\mathrm{C}=\mathrm{O}), 1606$ (N-C=O str), 737 (C-S str); ${ }^{1} \mathrm{H}-\mathrm{NMR}$ (DMSO-d ${ }_{6}$, $400 \mathrm{MHz}) \delta: 11.67(\mathrm{~s}, 1 \mathrm{H}), 7.63-7.60(\mathrm{dd}, J=8.4 \mathrm{~Hz}, 2.4 \mathrm{~Hz}, 1 \mathrm{H}), 7.59(\mathrm{~d}, J=2.4 \mathrm{~Hz}, 1 \mathrm{H})$, 7.44-7.35 (m, 5H), 7.34-7.31 (dd, $J=8.8 \mathrm{~Hz}, 2.4 \mathrm{~Hz}, 1 \mathrm{H}), 7.19-7.18(\mathrm{~d}, J=2.4 \mathrm{~Hz}, 1 \mathrm{H})$, 6.99-6.97 (d, $J=8.8 \mathrm{~Hz}, 1 \mathrm{H}), 6.93-6.90(\mathrm{~d}, J=8.8 \mathrm{~Hz}, 1 \mathrm{H}), 3.697(\mathrm{~s}, 2 \mathrm{H}) ;{ }^{13} \mathrm{C}$ NMR (DMSO$\left.\mathrm{d}_{6}, 100 \mathrm{MHz}\right) \delta$ : 196, 184, 174, 111-140, 78, 34; Anal. Found: C, 53.77; H, 2.75; N, 5.45; S, 6.24(\%). Calc. for $\left(\mathrm{C}_{23} \mathrm{H}_{14} \mathrm{ClBrN}_{2} \mathrm{O}_{3} \mathrm{~S}\right)$ : C, 53.71; H, 2.72; N, 5.44; S, 6.23(\%).

3'-(2-Benzoyl-4-chlorophenyl)-5-chloro-4'H-spiro[indole-3,2'-[1,3]thiazolidine]2,4'(1H)-dione; $($ D03)

M.p: $218^{0} \mathrm{C}$; IR (KBr) $\lambda_{\max }$ in $\mathrm{cm}^{-1}: 3201$ (N-H str), 3062 (Ar C-H str), 2962 (Ali C-H Str), 1696 (C=O str), 1671 (Spiro C=O), 1607 (N-C=O str), 733 (C-S str); ${ }^{1} \mathrm{H}-\mathrm{NMR}$ (DMSO-d 6 , $400 \mathrm{MHz}$ ) $\delta: 11.07(\mathrm{~s}, 1 \mathrm{H}), 7.76(\mathrm{~d}, J=2.4 \mathrm{~Hz}, 1 \mathrm{H}), 7.71-7.69(\mathrm{dd}, J=8.8 \mathrm{~Hz}, 2.4 \mathrm{~Hz}, 1 \mathrm{H}), 7.48-7.36(\mathrm{~m}$, $5 \mathrm{H}), 7.32-7.30(\mathrm{dd}, J=8.8 \mathrm{~Hz}, 2.4 \mathrm{~Hz}, 1 \mathrm{H}), 7.19-7.18(\mathrm{~d}, J=2.4 \mathrm{~Hz}, 1 \mathrm{H}), 7.0 \quad(\mathrm{~d}, J=8.8 \mathrm{~Hz}$, $1 \mathrm{H}), 6.86-6.84(\mathrm{~d}, J=8.8 \mathrm{~Hz}, 1 \mathrm{H}), 3.769(\mathrm{~s}, 2 \mathrm{H}) ;{ }^{13} \mathrm{C}-\mathrm{NMR}$ (DMSO-d $\left.6,100 \mathrm{MHz}\right) \delta: 196,184$, 174, 111-141, 78, 35; Anal. Found: C, 58.86; H, 2.91; N, 5.97; S, 6.83(\%). Calc. for $\left(\mathrm{C}_{23} \mathrm{H}_{14} \mathrm{Cl}_{2} \mathrm{~N}_{2} \mathrm{O}_{3} \mathrm{~S}\right)$ : C, 58.80; H, 2.98; N, 5.96; S, $6.83(\%)$.

3'-(2-Benzoyl-4-chlorophenyl)-5-nitro-4'H-spiro[indole-3,2'-[1,3]thiazolidine]2,4'(1H)-dione; (D04)

M.p: $211^{\circ} \mathrm{C}$; IR (KBr) $\lambda_{\max }$ in cm $\mathrm{cm}^{-1}: 3379$ (N-H Str), 3062 (Ar C-H Str), 2924 (Ali C-H Str), 1705 (C=O str), 1663 (Spiro C=O), 1616 (N-C=O str), 732 (C-S str); ${ }^{1} \mathrm{H}-\mathrm{NMR}$ (DMSO-d ${ }_{6}$, $400 \mathrm{MHz}) \delta$ : $11.45(\mathrm{~s}, 1 \mathrm{H}), 8.24-8.22(\mathrm{dd}, J=8.4 \mathrm{~Hz}, 2.4 \mathrm{~Hz}, 1 \mathrm{H}), 7.83-7.82(\mathrm{~d}, J=2.4 \mathrm{~Hz}$, $1 \mathrm{H}), 7.74-7.72(\mathrm{~d}, J=8.4 \mathrm{~Hz}, 1 \mathrm{H}), 7.51-7.33(\mathrm{~m}, 5 \mathrm{H}), 7.31-7.29(\mathrm{dd}, J=8.8 \mathrm{~Hz}, 2.4 \mathrm{~Hz}$, $1 \mathrm{H}), 7.19-7.18(\mathrm{~d}, J=2.4 \mathrm{~Hz}, 1 \mathrm{H}), 6.91-6.88(\mathrm{~d}, J=8.8 \mathrm{~Hz}, 1 \mathrm{H}), 3.758(\mathrm{~s}, 2 \mathrm{H}) ;{ }^{13} \mathrm{C} \mathrm{NMR}$ $\left(\mathrm{DMSO}_{6}, 100 \mathrm{MHz}\right) \delta: 196,182,174,112-144,78,34$; Anal. Found: C, 57.51; H, 2.91; N, 8.75; S, 6.68(\%). Calc. for $\left(\mathrm{C}_{23} \mathrm{H}_{14} \mathrm{ClN}_{3} \mathrm{O}_{5} \mathrm{~S}\right)$ : C, 57.57; H, 2.94; N, 8.76; S, 6.68(\%). 
3'-[4-Chloro-2-(phenylcarbonyl)phenyl]-5'-methyl-4'H-spiro[indole-3,2'-[1,3] thiazolidine]-2, 4' (1H)-dione; (E01)

M.p: $234{ }^{\circ} \mathrm{C}$; IR (KBr) $\lambda_{\max }$ in $\mathrm{cm}^{-1}$ : 3209 (N-H Str), 3055 (Ar C-H Str), 2924, 2877 (Ali C-H Str), 1705 (C=O str), 1672 (Spiro $\mathrm{C}=\mathrm{O}), 1604$ ( $\mathrm{N}-\mathrm{C}=\mathrm{O}$ str), 728 (C-S str); ${ }^{1} \mathrm{H}-\mathrm{NMR}$ $\left(\right.$ DMSO-d $\left._{6}, 400 \mathrm{MHz}\right) \delta: 11.56(\mathrm{~s}, 1 \mathrm{H}), 7.73-7.69(\mathrm{t}, J=8.4 \mathrm{~Hz}, 1 \mathrm{H}), 7.64-7.62(\mathrm{~d}, J=7.6$ $\mathrm{Hz}, 1 \mathrm{H}), 7.52-7.37$ (m, 5H), 7.33-7.30 (dd, $J=8.8 \mathrm{~Hz}, 2.4 \mathrm{~Hz}, 1 \mathrm{H}), 7.29-7.25(\mathrm{~d}, J=7.6 \mathrm{~Hz}$, $1 \mathrm{H}), 7.18-7.17(\mathrm{~d}, J=2.4 \mathrm{~Hz}, 1 \mathrm{H}), 7.07-7.03(\mathrm{t}, J=7.6 \mathrm{~Hz}, 1 \mathrm{H}), 6.93-6.91(\mathrm{~d}, J=8.8 \mathrm{~Hz}$, $1 \mathrm{H}), 3.87-3.81(\mathrm{q}, J=7.2 \mathrm{~Hz}, 1 \mathrm{H}), 1.38-1.37$ (d, $J=7.2 \mathrm{~Hz}, 3 \mathrm{H}) ;{ }^{13} \mathrm{C}-\mathrm{NMR}$ (DMSO-d 6,100 MHz) $\delta:$ 196, 184, 178, 110-141, 79, 49, 19; Anal. Found: C, 64.21; H, 3.82; N, 6.24; S, 7.14 (\%). Calc. for $\left(\mathrm{C}_{24} \mathrm{H}_{17} \mathrm{ClN}_{2} \mathrm{O}_{3} \mathrm{~S}\right): \mathrm{C}, 64.15 ; \mathrm{H}, 3.78 ; \mathrm{N}, 6.23 ; \mathrm{S}, 7.14(\%)$.

5-Bromo-3'-[4-chloro-2-(phenylcarbonyl)phenyl]-5'-methyl-4'H-spiro[indole-3,2'[1,3] thiazolidine]-2,4'(1H)-dione; (E02)

M.p: $225{ }^{\circ} \mathrm{C}$; IR (KBr) $\lambda_{\max }$ in $\mathrm{cm}^{-1}: 3417$ (N-H Str), 3062 (Ar C-H Str), 2924, 2877 (Ali C-H Str), 1705 (C=O str), 1661 (Spiro C=O), 1612 (N-C=O str), 740 (C-S str); ${ }^{1} \mathrm{H}-\mathrm{NMR}$ $\left(\right.$ DMSO-d $\left._{6}, 400 \mathrm{MHz}\right) \delta: 11.59$ (s, $\left.1 \mathrm{H}\right), 7.69-7.66(\mathrm{dd}, J=8.4 \mathrm{~Hz}, 2.4 \mathrm{~Hz}, 1 \mathrm{H}), 7.64-7.63$ (d, $J$ $=2.4 \mathrm{~Hz}, 1 \mathrm{H}), 7.49-7.37(\mathrm{~m}, 5 \mathrm{H}), 7.35-7.32(\mathrm{dd}, J=8.8 \mathrm{~Hz}, 2.4 \mathrm{~Hz}, 1 \mathrm{H}), 7.18-7.17(\mathrm{~d}, J$ $=2.4 \mathrm{~Hz}, 1 \mathrm{H}), 6.93-6.91(\mathrm{~d}, J=8.8 \mathrm{~Hz}, 1 \mathrm{H}), 3.61-3.55(\mathrm{q}, J=7.2 \mathrm{~Hz}, 1 \mathrm{H}), 1.35-1.33(\mathrm{~d}, J$ $=7.6 \mathrm{~Hz}, 3 \mathrm{H}) ;{ }^{13} \mathrm{C}-\mathrm{NMR}\left(\mathrm{DMSO}_{6}, 100 \mathrm{MHz}\right) \delta$ : 196, 184, 178, 110-141, 80, 49, 19; Anal. Found: C, 54.61; H, 3.06; N, 5.31; S, 6.07(\%). Calc. for $\left(\mathrm{C}_{24} \mathrm{H}_{16} \mathrm{ClBrN}_{2} \mathrm{O}_{3} \mathrm{~S}\right): \mathrm{C}, 54.56 ; \mathrm{H}$, $3.03 ; \mathrm{N}, 5.30 ; \mathrm{S}, 6.07(\%)$.

5-Chloro-3'-[4-chloro-2-(phenylcarbonyl)phenyl]-5'-methyl-4'H-spiro[indole-3,2'[1,3 ]thiazolidine]-2,4'(1H)-dione; (E03)

M.p: $212{ }^{\circ} \mathrm{C}$; IR (KBr) $\lambda_{\max }$ in $\mathrm{cm}^{-1}: 3417$ (N-H Str), 3062 (Ar C-H Str), 2924, 2877 (Ali CH Str), 1705 (C=O str), 1664 (Spiro $\mathrm{C}=\mathrm{O}$ ), 1612 (N-C=O str), 731 (C-S str); ${ }^{1} \mathrm{H}-\mathrm{NMR}$ $\left(\right.$ DMSO-d $\left._{6}, 400 \mathrm{MHz}\right) \delta: 11.05(\mathrm{~s}, 1 \mathrm{H}), 7.64-7.61(\mathrm{dd}, J=8.4 \mathrm{~Hz}, 2.4 \mathrm{~Hz}, 1 \mathrm{H}), 7.59-7.52$ (d, $J=2.4 \mathrm{~Hz}, 1 \mathrm{H}), 7.42-7.35(\mathrm{~m}, 5 \mathrm{H}), 7.32-7.29(\mathrm{dd}, J=8.8 \mathrm{~Hz}, 2.4 \mathrm{~Hz}, 1 \mathrm{H}), 7.19-7.18(\mathrm{~d}, J$ $=2.4 \mathrm{~Hz}, 1 \mathrm{H}), 6.91-6.89(\mathrm{~d}, J=8.8 \mathrm{~Hz}, 1 \mathrm{H}), 6.84-6.82(\mathrm{~d}, J=8.8 \mathrm{~Hz}, 1 \mathrm{H}), 3.49-3.44(\mathrm{q}, J$ $=6.8 \mathrm{~Hz}, 1 \mathrm{H}), 1.32-1.31(\mathrm{~d}, J=7.6 \mathrm{~Hz}, 3 \mathrm{H}) ;{ }^{13} \mathrm{C}-\mathrm{NMR}\left(\mathrm{DMSO}_{6}, 100 \mathrm{MHz}\right) \delta: 196,184$, 178, 111-141, 79, 49, 18; Anal. Found: C, 59.64; H, 3.34; N, 5.80; S, 6.63(\%). Calc. for $\left(\mathrm{C}_{24} \mathrm{H}_{16} \mathrm{Cl}_{2} \mathrm{~N}_{2} \mathrm{O}_{3} \mathrm{~S}\right)$ : C, 59.58; H, 3.31; N, 5.79; S, 6.63(\%).

3'-[4-Chloro-2-(phenylcarbonyl)phenyl]-5'-methyl-5-nitro-4'H-spiro[indole-3,2'[1,3]thiazolidine]-2,4'(1H)-dione; (E04)

M.p: $236{ }^{\circ} \mathrm{C}$; IR (KBr) $\lambda_{\max }$ in $\mathrm{cm}^{-1}: 3379$ (N-H Str), 3062 (Ar C-H Str), 2924, 2854 (Ali C-H Str), 1705 (C=O str), 1641 (Spiro C=O), 1607 (N-C=O str), 732 (C-S str); ${ }^{1} \mathrm{H}-\mathrm{NMR}$ $\left(\right.$ DMSO-d $\left._{6}, 400 \mathrm{MHz}\right) \delta: 11.68(\mathrm{~s}, 1 \mathrm{H}), 8.64-8.61(\mathrm{dd}, J=8.8 \mathrm{~Hz}, 2.4 \mathrm{~Hz}, 1 \mathrm{H}), 7.94(\mathrm{~d}, J$ $=2.4 \mathrm{~Hz}, 1 \mathrm{H}), 7.50-7.35(\mathrm{~m}, 5 \mathrm{H}), 7.32-7.29(\mathrm{dd}, J=8.8 \mathrm{~Hz}, 2.4 \mathrm{~Hz}, 1 \mathrm{H}), 7.19-7.18(\mathrm{~d}, J$ $=2.4 \mathrm{~Hz}, 1 \mathrm{H}), 6.92-6.89(\mathrm{~d}, J=8.8 \mathrm{~Hz}, 1 \mathrm{H}), 3.49-3.43(\mathrm{q}, J=7.2 \mathrm{~Hz}, 1 \mathrm{H}), 1.32-1.31(\mathrm{~d}, J$ $=7.6 \mathrm{~Hz}, 1 \mathrm{H}) ;{ }^{13} \mathrm{C}-\mathrm{NMR}\left(\mathrm{DMSO}_{6}, 100 \mathrm{MHz}\right) \delta$ : 196, 183, 178, 109-144, 79, 49, 19; Anal. Found: C, 58.36; H, 3.26; N, 8.51; S, 6.50(\%). Calc. for $\left(\mathrm{C}_{24} \mathrm{H}_{16} \mathrm{ClN}_{3} \mathrm{O}_{5} \mathrm{~S}\right): \mathrm{C}, 58.31 ; \mathrm{H}$, $3.23 ; \mathrm{N}, 8.50 ; \mathrm{S}, 6.49(\%)$.

\section{Antimicrobial activity}

In vitro antibacterial activity was determined by Kirby-Bauer disc diffusion method against bacteria such as Staphylococcus aureus and Bacillus (Gram +ve), Salmonella typhi and 
Pseudomonas aeruginosa (Gram -ve) using Ampicilin as a standard drug. The standard and test compounds were prepared in DMSO at different concentrations. The zone of inhibition was compared with standard drug after $24 \mathrm{~h}$ incubation at $35-37^{\circ} \mathrm{C}$.

Similarly antifungal activity was performed against Candida. The standard and test compounds were prepared in DMSO at different concentrations. The zone of inhibition was compared with standard drug after $48 \mathrm{~h}$ at $25{ }^{\circ} \mathrm{C}$. The results of antibacterial and antifungal activity are presented in Table 1.

Table 1. Antimicrobial activity of the synthesized compounds Zone of inhibition (mm) of synthesized compounds

\begin{tabular}{|c|c|c|c|c|c|c|c|c|c|c|c|c|c|c|c|c|c|c|c|c|}
\hline \multirow{4}{*}{ 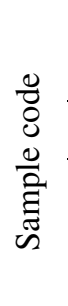 } & \multicolumn{16}{|c|}{ Anti-bacterial activity } & \multirow{2}{*}{\multicolumn{4}{|c|}{$\begin{array}{l}\text { Anti-fungal } \\
\text { activity }\end{array}$}} \\
\hline & \multicolumn{8}{|c|}{ Gram positive } & \multicolumn{8}{|c|}{ Gram negative } & & & & \\
\hline & \multicolumn{4}{|c|}{ Staphylococcus.spp } & \multicolumn{4}{|c|}{ Bacillus.spp } & \multicolumn{4}{|c|}{ Salmonella.spp } & \multicolumn{4}{|c|}{ Pseudomonas.spp } & \multicolumn{4}{|c|}{ Candida } \\
\hline & $\begin{array}{l}\circlearrowright_{\Xi}^{\infty} \\
\stackrel{\Xi}{8}\end{array}$ & $\begin{array}{l}\stackrel{0}{0} \\
\stackrel{8}{8} \\
\stackrel{8}{n}\end{array}$ & $\begin{array}{l}\stackrel{0}{0} \\
\stackrel{d}{d} \\
\end{array}$ & $\vec{D}$ & $\begin{array}{l}\text { b } \\
\text { ఏ } \\
8 \\
8\end{array}$ & 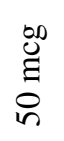 & 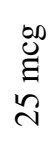 & $\vec{D}$ & $\begin{array}{l}0 \\
\stackrel{0}{0} \\
8 \\
\varnothing\end{array}$ & $\begin{array}{l}\stackrel{b}{0} \\
\stackrel{8}{8} \\
\stackrel{8}{n}\end{array}$ & $\begin{array}{l}\stackrel{b 0}{0} \\
\Xi \\
\end{array}$ & $\vec{D}$ & $\begin{array}{l}b_{0}^{0} \\
\stackrel{\Xi}{8} \\
\stackrel{0}{8}\end{array}$ & $\begin{array}{l}\stackrel{\infty}{\Xi}^{\prime} \\
\stackrel{8}{n}\end{array}$ & $\begin{array}{l}\stackrel{00}{\Xi} \\
\stackrel{d}{c}\end{array}$ & $\vec{\nabla}$ & $\begin{array}{l}\stackrel{\infty}{0} \\
\Xi \\
8 \\
\varrho\end{array}$ & 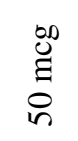 & 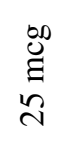 & $\vec{\nabla}$ \\
\hline D01 & 07 & 02 & - & 11 & 02 & 03 & - & 10 & 05 & 02 & - & 10 & 08 & 05 & 02 & 11 & 16 & 09 & 02 & 21 \\
\hline D02 & 06 & 05 & 03 & 11 & 08 & 07 & 06 & 09 & 08 & 05 & 02 & 10 & 05 & 02 & - & 10 & 07 & 04 & 02 & 15 \\
\hline D03 & 06 & 03 & 03 & 10 & 08 & 06 & 04 & 10 & 07 & 04 & 02 & 10 & 08 & 05 & 02 & 11 & 19 & 13 & 10 & 21 \\
\hline D04 & 07 & 06 & 05 & 11 & 07 & 06 & 02 & 09 & 07 & 05 & 03 & 09 & 07 & 04 & 03 & 11 & 17 & 11 & 08 & 21 \\
\hline E01 & 07 & 06 & 03 & 08 & 07 & 05 & 02 & 10 & 09 & 06 & 02 & 11 & 09 & 06 & 03 & 12 & 10 & 06 & 04 & 20 \\
\hline E02 & 09 & 06 & 04 & 12 & 10 & 07 & 03 & 11 & 07 & 05 & 02 & 09 & 08 & 05 & 02 & 12 & 10 & 07 & 03 & 14 \\
\hline E03 & 08 & 05 & 03 & 10 & 09 & 05 & 02 & 11 & 09 & 05 & - & 11 & 09 & 06 & 03 & 10 & 17 & 09 & 05 & 20 \\
\hline E04 & 07 & 04 & 02 & 11 & 06 & 02 & - & 12 & 07 & 04 & - & 10 & 08 & 04 & 02 & 11 & 18 & 10 & 07 & 21 \\
\hline
\end{tabular}

The compounds of D01-D04 and E01-E04 showed significant activity against selected bacteria. Antifungal activity was performed on candida. The compounds D02, E02, E03 and D04 showed moderate activity against the fungus. The compound D03 was more active among screened compounds against Candida.

\section{Conclusion}

The reaction profile explained in the present work is very efficient to synthesis, characterization and antimicrobial evaluation of $5-\mathrm{Br}, 5-\mathrm{Cl}$ and $5-\mathrm{NO}_{2}$ indole-2,3-dione based spiro-4-thiazolidiones. The prepared compounds showed significant and moderate antimicrobial activities and these are promising compounds for further pharmacological studies.

\section{References}

1. Sannigrahi M, Tetrahedron, 1999, 55(30), 9007-9071; DOI:10.1016/S00404020(99)00482-2

2. James D M, Kunze H B and Faulkner D J, J Nat Prod., 1991, 54(4), 1137-1140; DOI:10.1021/np50076a040

3. Kobayashi J, Tsuda M, Agemi K, Shigemiri H, Ishibashi M, Sasaki T and Mikami Y, Tetrahedron, 1991, 47(33), 6617-6622; DOI:10.1016/S0040-4020(01)82314-0

4. De Silva J F M, Garden S J and Pinto A C, J Braz Chem Soc., 2001, 12(3), 273.

5. Erdmann O L, J Prakt Chem., 1840, 19(1), 321-362; DOI:10.1002/prac.18400190161 
6. Laurent A, Ann Chim Phys., 1840, 3, 372.

7. Pandeya S N, Sriram D, Nath G and Clercq E De, Eur J Pharm Sci., 1999, 9(1), 25-

31; DOI:10.1016/S0928-0987(99)00038-X

8. Pandeya S.N, Sriram D, Nath G and Clercq E De, IL Farmaco., 1999, 54(9), 624-628; DOI:10.1016/S0014-827X(99)00075-0

9. Meenakshi K, Sammaiah G, Sarangapani M and Venkateswar Rao J, Indian J Heterocycl Chem., 2006, 16, 21-24.

10. Dilber S, Saban M, Gelinco A, Arsenijevi L, Bogavac M and Pavlov S, Pharmazie, 1990, 45, 800-805.

11. Singh R.V, Fahmi N and Biyala M.K, J Iranian Chem Soc., 2005, 2, 40-46; DOI:10.1007/BF03245778

12. Panwar H, Verma R S, Srivastava V K and Kumar A, Indian J Chem., 2006, 45(B), 2099-2104.

13. Sridhar S K, Pandeya S N, Stables J P and Ramesh A, Eur J Pharm Sci., 2002, 16(3), 129-132; DOI:10.1016/S0928-0987(02)00077-5

14. Pandeya S N and Raja A S, J Pharm Pharm Sci., 2002, 5(3), 266-271.

15. Sridhar S K and Ramesh A, Biol Pharm Bull., 2001, 24(10), 1149-1152;

DOI:10.1248/bpb.24.1149

16. Srivastava S. K, Srivastava S and Srivastava S D, Indian J Chem., 1999, 38(B) 183-187.

17. Brana M F, Gradillas A, et al., J Med Chem., 2004, 47(9), 2236-2242; DOI:10.1021/jm0310784

18. Popp F D, Randy Parson and Pajouhesh H, J Pharm Sci., 1983, 72(3), 318-321; DOI:10.1002/jps.2600720330

19. Varma R S and Pandeya R K, Indian J Pharm Sci., 1982, 46, 132-135.

20. Webber S E, Tikhe J, Worland S T, Fuhrman S A, Hendrickson T F, Mathews D A, Love R A, Patick A K et al., J Med Chem., 1996, 39(26), 5072-5076;

DOI:10.1021/jm960603e

21. Medvedev A E, Goodwin A, Clow A, Halket J, Glover V and Sandler M, Biochem Pharmacol., 1992, 44(3), 590-592; DOI:10.1016/0006-2952(92)90454-Q

22. Varma R S, Prakash R and Abid Ali Khan M M, Indian Drugs, 1986, 23(16), 345-349.

23. Selvam P, Chandramohan M, Clercq E De, Witvrouw M and Pannecouque C, Eur J Pharm Sci., 2001, 14(4), 313-316; DOI:10.1016/S0928-0987(01)00197-X

24. Kavitha C V, Basappa S, Nanjunda S, Mantelingu K, Doreswamy S, Sridhar M A, Prasad J S and Rangappa K S, Bioorg Med Chem., 2006, 14(7), 2290-2299; DOI:10.1016/j.bmc.2005.11.017

25. Ottana R, Maccari R, Barreca M L, Bruno G, Rotondo A, Rossi A, Chiricosta G, Di Paola R, Sautebin L, Cuzzocrea S and Vigorita M G, Bioorg Med Chem., 2005, 13(13), 4243-4252; DOI:10.1016/j.bmc.2005.04.058

26. Kucukguzel G, Kocatepe A, Clercq E. De, Sahin F and Gulluce M, Eur J Med Chem., 2006, 41(3), 353-359; DOI:10.1016/j.ejmech.2005.11.005

27. Jain S C, Sinha J, Bhagat S, Errington W and Olsen C E, Synth Commun., 2003, 33, 563-577; DOI:10.1081/SCC-120015810

28. Migilaiah K and Babu R R, Indian J Chem., 1998, 37(B), 894.

29. Zahir Hussain A and Nagoor Meeran M, RJPBCS, 2015, 6(1), 1598-1601.

30. Mashelkar Uday C, Rane Deepak M, Indian J Chem., 2005, 44(B), 1937-1939. 